\title{
Understanding the 'Electronic' Student: Analysis of Functional Requirements for Distributed Education
}

\author{
Linda Carswell, Pete Thomas, Marian Petre, Blaine Price, Mike Richards \\ Centre for Informatics Education Research, \\ Computing Department, The Open University, UK \\ E-mail: 1.carswell@open.ac.uk
}

\begin{abstract}
This paper describes how the Open University, as a large distance education institution, has used the Internet to transform the learning environment for distance students. We review the process involved in understanding the requirements of distance education students and how they can be supported via the Internet. We describe the tools developed in our Internet-based implementation and how they address the needs of geographically remote learners: electronic student registration, electronic assignment handling, electronic tutorial sessions, and electronic examinations. The paper reflects on the impact of this implementation and the gains in using the Internet for global distance learners, realised in flexible study practices and efficient communiqués.
\end{abstract}

\section{KEYWORDS}

Internet distance education, Virtual campus, Distance learning, Distance teaching, Electronic student

\section{INTRODUCTION}

The Internet is often portrayed as a cure all for education and society. However, we need to understand how to use it in order to exploit its potential. An essential requirement is an understanding of our students and what aspects of the Internet can support them. An unfortunate trap has been the translation of existing material and practices into alternative medium, with little gain. For example, what does the student gain from having lecture notes on the web: is it lecture replacement or lecture aid, and is it appropriate? We need to ensure that the purpose we intend to use the Internet for is the one we are actually using it for: is the Internet supporting student needs or technology vanity?

Universities naturally want to respond to the latest technology - the Internet [1]. They too see the advantages of global, accessible information resources [2], while anticipating (realistically or otherwise) technological solutions to increasing student-staff ratios, diminishing funding, and improving student experiences [3], [4], [5].

The Internet is an important advance for distance education. It has the potential to meet students' changing needs - in particular choice of their own time and place, of study. We want to offer students a virtual campus addressing these issues and the isolation that many students endure. OU students are based off-campus remote from tutors (part-time teachers or part-time associate lecturers), fellow students, and university facilities, and can never realistically attend lectures. So our aim is not to replace, but to augment what already exists. 
However, we need to guard against ill-considered technological solutions. Simple translations from familiar media into electronic media are rarely productive. They are certainly inadequate for supported distance education, which aims to engage students in a community of learning. If we hope to improve rather than translate, we must examine our motives critically and recognise what the potential costs and gains may be, such as:

- What is driving the change: need or trend?

- Who is driving the change: student or institution?

- Who will gain?

- Who will pay?

\section{DISTANCE LEARNING AT THE OPEN UNIVERSITY}

In order to understand the reasoning behind our Internet-based implementation it is important to put in context the OU distance education model and profile the OU student. We make distinction here because confusion surrounds the term distance education. We shall assume that distance education includes distance learning and distance teaching rather than drawing out the distinctions in this paper [6]. It would be inappropriate to assume that the exemplar described here will work for all distance education students.

The term distance education conjures up different models and interpretation depends on culture, scale and operational procedures [7]. The North American model is characteristically technological [8] where students receive satellite or video linked lectures, designed to solve a resource issue rather than addressing flexible student-centred study. The instructor, not student, is remote from the campus and the educational experience differs little from that of conventional students.

Other models, often of limited scope, make traditional materials available electronically for remote students, offered as self study materials. However, these were not designed as such and typically fail to address the specific and different needs of distance learners. Some models have encouraged relatively small groups of students to submit work electronically to their instructor for on-screen marking, but again, this is usually with campus based students [9]. These models still impose constraints upon the learner in terms of time and place.

In contrast, the Open University distance education model has been designed for adults studying part-time at home who are unlikely to have had recent formal education. Such students are typically employed full-time and have family or other responsibilities with which their studies have to merge. The university offers courses in the majority of subject disciplines. Courses include components such as:

- Specifically designed distance educational course materials

- Video and audio cassettes

- Television broadcasts

- Home experimental kits that 'turn a kitchen into a laboratory'

- A personal tutor who provides academic support, i.e., Marking and commenting on assignments, answering student queries, and providing FTF teaching sessions

- Short (week long) residential schools

- Educational software

- Assignments 
- A final (written) supervised examination

OU students typically:

- Study part time

- Are remote from university campus and its resources (library, laboratory)

- Are remote from university teachers

- Are remote from other students

- May not have formal educational qualifications

OU's distance learning relies on course materials that have a dual role:

- To provide all necessary course content, and

- To foster self-study skills

However, teaching and support primarily occurs when the tutor marks a written assignment (known internally as Tutor Marked Assignments (TMAs)). This has two purposes: assessing student progress, and teaching. To ensure assessment quality and consistency for the student, tutor notes (including a marking scheme) are provided, and marked TMAs are regularly monitored. On a course with five thousand students (e.g., an introductory computing course) approximately 200 part-time tutors' work must be co-ordinated and standardised.

The OU's approach to distance education is learner-centred--“supported open learning"-characterised by:

- Open and equal access: It has no barriers to entry, and courses are designed to allow students with little formal background to reach university entrance level quickly.

- Commitment to quality: All courses undergo advanced rigorous developmental testing before presentation, and during presentation all TMA marking is monitored and coordinated at a uniform standard. (Some courses have up to 9,000 students.)

- Support for students: Students are provided with rapid feedback and with counselling for academic and personal study problems in order to retain and motivate them. (This makes communication - paper-based or otherwise — a key issue.)

Despite this mission, students can still be disadvantaged: personal, local, or geographical constraints may restrict individual students' access to FTF sessions or telephone contact with the tutor and the university. This predominately paper-based system, while proving itself to be a reliable model within the UK (which has one of the fastest and most reliable postal systems in the world), has disadvantages. It lacks the desired flexibility and rapidity of communication important for sustaining distance students, especially outside the UK. These are barriers to supporting the any time, any place OU model, especially those outside the UK. An Internetbased system is an obvious solution; however it must prove itself secure, robust, scaleable, and affordable in order to be an acceptable model for the university to adequately support a volume of distance education students. 


\section{THE REQUIREMENTS OF A DISTANCE EDUCATION STUDENT}

In understanding the issues for distance education students and how they might be supported in an Internet-based implementation, it was necessary to become the remote student and attempt to register and study with the university. We were keen to experience how the OU presents itself to its clients/students; we chose a location as remote as was feasible to see the effects of distance: Canada. This represented significant challenges in distance, time zones, and postal communication. In the trial, the remote student exercise showed that there were difficulties with:

- Finding information

- Speed of the local postal service

- Speed of student registration

- Speed of course enrolment

Students who are geographically remote suffer obvious time delays in the paper-based system. While electronic communication seems a straightforward answer for such communiqués, the university does not have a process to deal with ad-hoc requests, as administrators work within a mechanised, scaleable, but paper-based model. This was stumbling block, and a seemingly simple replacement by an Internet-based implementation would not be sufficient.

Even the choice of implementing electronic communication systems presents problems depending on the location of the student. Some countries have free local calls while others have per-minute telephone charges on every call, which discourages unlimited online synchronous access due to the cost. However cost is not the only factor. We tried using SoftArc's First Class synchronous electronic conferencing with a client in Toronto accessing a server in the UK, but the tens of seconds latency between commands being sent and executed made the synchronous interaction unusable. Such latency can also occur on a busy network within a continent depending on the position of one's ISP, so it is clear that both synchronous and asynchronous communication models are required in order to address the issues of cost and latency.

\section{REQUIREMENTS ANALYSIS}

We used the experiences from the remote student exercise to conduct an initial pilot with 30 volunteer students (all of whom were outside Europe). They provided logs of their interactions and completed questionnaires evaluating the delivery and interaction mechanisms. The experiences from both of these exercised prompted a thorough analysis of four areas:

- The registration system: registration and general administrative process

- The electronic assignment handling system: submission, marking and return of assignments

- Student academic queries: support for student queries and tutorials

- The examination process

While our goal was to provide an Internet-based system giving an improved service for our students, it was clear that change in one area had repercussions in another. While the 25-year-old paper-based system runs like a finely tuned machine, capable of processing large numbers of students efficiently, it fails to translate directly into an Internet-based model. Some additional rationalisation was required. We shall examine each area in turn. 


\section{A. The Registration System}

Annual enrolment is a two-stage process: first, students reserve a place on their selected course(s) and receive course information; second, they register for their chosen course(s). In the paperbased registration process, students write, phone, or fax, the University. If students are within the same country or time zone this is not problematic, but for students who are geographically remote, the interactions are time dependent, resulting in obstacles to enrolment.

\section{B. The Electronic Assignment Handling System}

In the paper-based model, students post their assignments to their tutor who marks and comments on the script. The assignment is returned to the student via the University. This is an effective and robust system due to the reliable and relatively quick UK postal service. However, the TMA (tutor marked assignment) is posted three times (sometimes four if it also goes to the monitor), delaying the return of an assignment for at least a week. Attempts at speeding up this paper-based system have had significant costs and introduce complexity, both of which prohibit scalability. As these are the main teaching instruments, improvements are of prime importance.

There are five major disadvantages with the paper system.

1. A student does not know if an assignment has been received

2. TMAs can get lost, and a student may not have photocopied their work as advised

3. The TMA turn-round time is lengthy and the impact of remedial advice is lost

4. Completion of the 4-part paper control form is time consuming and error prone and results in significant and costly manual remedial action

5. The reliability of the UK postal system is not replicated in all countries, making it difficult for students in some countries to study effectively.

\section{Student Academic Queries}

An important feature of supported open learning is the student's interaction with the tutor, who provides academic support to approximately 25 students. There are two strands to this association: one-to-one interaction and FTF tutorials.

\section{One-to-one Interaction}

Typical interactions include tutors answering academic queries and providing general help and support (most usually via telephone). As students require timely help, the inability to contact a tutor at the crucial moment can result in learning delays and student frustration. This has the implicit restriction of the same time, any place model, where tutors are only available at particular times—not necessarily convenient for the student.

\section{Face-to-face Tutorials}

The tutor meets the students FTF at a tutorial session which covers a number of disparate activities: group working, resolving student problems, facilitating problem-solving, and targeting interactive tasks to reinforce taught material. There are also implicit benefits such as studentstudent interaction and peer group teaching and assessment. While such interactions are desirable, experience has shown that many students have difficulty attending FTF tutorials, often because of their location but also because of other competing commitments. Tutorials are not without their costs: both considerable administration to organise local student-tutor allocations near a study centre and study centre procurement are costly. Either of these issues is reason enough to seek alternative viable solutions. 


\section{The Examination}

To verify TMA grades students must sit a closed-book final exam under strict supervision. For students outside the UK special arrangements have to be made which can be quite costly. Obvious time delays can be incurred with the registered delivery of the exam paper and the written script to and from the university. This assessment model suffers from the constraints of the same time, same place model and could benefit from faster but secure transmission of exam paper and student script.

\section{THE INTERNET-BASED IMPLEMENTATION}

Analysis of the above areas illustrated the problems associated with the paper-based model. The real challenge for electronic communication is not just to translate what already exists but to address the deficiencies highlighted. Although we considered the needs of other participants (tutors and administrators) in this learning process, this paper focuses on student requirements. The Internet-based implementation makes significant gains in each of the following areas:

\section{A. Electronic Registration}

An automatic Web registration system was designed to support students who required increased flexibility and who were geographically remote, requiring the immediacy of Internet transmission and acknowledgement. Students can now reserve a place for a course via the Internet using a Web form (see http://www3.open.ac.uk/courses/information/online.htm). The Web form enables electronic data capture which has positive implications for scalability, improving efficiency for the student and for university resources. It also enables the student to enter data on a 'smart' form that insists on required fields being completed. This data is verified before allowing the form to be submitted, saving unnecessary time delays due to information gaps. This automates the registration system. Until recently, all student information was captured on paper and entered manually into a database.

\section{B. The Electronic Assignment Handling System}

The Unix-based electronic assignment handling system, with supporting software written in Perl and Java, involves a centralised collection of databases. The system allows a student to submit a TMA electronically and receive the marked TMA electronically.

\section{Student TMA Submission}

The student submits a word-processed TMA document via a secure Web form to a central database. The arrival of a TMA immediately causes two actions to be taken:

a) A unique receipt number is sent to the student acknowledging the received TMA. This is a useful audit mechanism not available in the postal system - the student is assured that their TMA has arrived.

b) An e-mail message informs the tutor of the TMA waiting to be downloaded onto their computer.

The system matches the student's personal identifier number with their details stored in a database that contains the student's unique identification number and related information. The tutor collects the student's TMA and downloads it to his or her machine for marking. The significance of this is the elimination of the error-prone administrative paper form-the student no longer has to complete a lengthy form, details on which are sources of error. 


\section{The Marked TMA}

The tutor marks and comments on the student's assignment electronically. The marking tool is a piece of software that provides various aids to on-screen commenting and grading. The marking tool performs local validation of marks and computes the overall grade, thereby eliminating another source of administrative error. The advantage to the student is the timely return of a correctly marked assignment with legible comments (a common complaint on paper assignments is the inability to read their tutor's handwriting).

The electronic assignment handling system has eliminated some disadvantages of the paper-based system and improved functionality. Student TMA submissions are acknowledged; copies of assignments are retained (not possible in the paper-based model); and turn-around time of TMA processing has been reduced, (the current record is 4 hours, typical turnaround time is 4-7 days - half the time for paper assignments). Gratifyingly, improvements stretch beyond our initial goals for the students: administrative processes such as TMA monitoring are instantaneously available; student progress is easily aggregated and made available to tutors; and turn-round time for assignments can be monitored. The electronic assignment submission system has improved the support given to students but more importantly has provided students with timely return of assignments which provides teaching and sustains motivation. The benefits to the student can be summarised as:

- The ability to submit a TMA from anywhere - without the inconvenience of posting their work

- No postal delays

- They retain a copy of their work on their machine

- They get a receipt for the work they have submitted, which is date and time stamped

- The student can read the tutor comments - they no longer have to grapple with illegible handwriting

- The turn-around time of TMAs is reduced

\section{Tutorial Sessions}

The two main types of interaction take the form of individual interactions between student and tutor or group interactions between tutor and students. We did experiment with synchronous interactions using video-conferencing tools such as $\mathrm{Cu}-\mathrm{See}-\mathrm{Me}$, but these had several restrictions. The technology was not stable enough to be reliable and useful. Bandwidth caused latency problems effecting both audio and video. Interestingly enough, the more useful features, had they been reliable, were a whiteboard and audio; video added little after the student had initially 'seen' the tutor. However, the current video conferencing software available needs more development before a reliable, affordable, off-the-shelf product would be viable enough to use for remote students. This synchronous model does have the advantages of immediacy but is hampered by the need for expensive equipment and the ability for students and tutors to meet at a specific time. The most successful mode of interaction for tutoring was asynchronous.

\section{One-to-one Interaction}

These interactions are supported by asynchronous e-mail. Flexibility is not the only factor in this exemplar. Students are required to formulate their problem into text, causing them to reflect upon their learning experience and formulate a textual representation of their problem. The queries received via e-mail are constructive questions demonstrating a deep reading and understanding of the material. Based on an analysis of a corpus of interactions of student problems, this interaction has fostered learning. 


\section{Tutorials: Group Problem Sessions}

The tool used to support group sessions was HyperNews, an asynchronous Web-based conferencing system, (with e-mail gateway), which most web browsers support. The students were able to post messages, which were threaded, and could get responses from both the tutor and fellow students. We observed students participating in peer-group teaching often resolving problems before the tutor had a chance to answer. Many students preferred to follow the interactions without being required to participate. The advantages for students included the ability to participate, the ability to observe, and the ready access to a community of learners.

The success of this tool is the ability to provide flexible, immediate communication that overcomes the barrier of geographic remoteness. Not only can we reach those students for whom attendance at FTF tutorials is difficult we can also offer our courses outside the UK.

\section{The Electronic Examination}

Having electronic examination poses some interesting problems for an institution. For a student it can allow the use of a keyboard and the ability to iterate a solution. Many students now wordprocess their assignments: they have key-board skills and practices associated with the flexibility of word-processing. For the institution the concern is the equality of opportunity and ensuring that cheating does not occur. We were able to convince the university to let us try such a venture with a small sample of geographically remote students to test its viability. The examination paper was downloaded as an encrypted document via a secure web page by the exam supervisor in an appointed exam centre. Once the exam had commenced the student was free to print the exam paper. On exam completion the student's exam script (keyed into a word processor) was encrypted and returned via a secure web page by the exam supervisor. The student was allowed to bring in his or her own keyboard if desired (similar to bringing a favourite pen). The supervisor's job was to ensure a clean machine (one without files that reference or pertain to course materials) with a network connection which was disabled during the exam. The advantage for students was the ability to take exams near their homes while using an input device with which they were familiar.

We are hoping to extend this work further this year. However, this will require some substantial rethinking of the assessment model, including a reappraisal of the underlying objectives of written examinations. Research is underway investigating novel forms of examination that can exploit the technology while providing the student with a testing mechanism that allows them to demonstrate their knowledge and understanding. So far, we have established that parts of the process can be managed effectively over the Internet with a resulting reduction in costs.

\section{EVALUATION OF THE CHANGES: THE STUDENT PERSPECTIVE}

In order to assess the impact of this Internet-based implementation we elicited the help of 300 students on two computing courses. We studied these students closely and gathered statistics on the demographics, types, frequency of interactions, assignment styles, as well as questionnaire responses. This information was used to evaluate the system in order to assess if such a venture was viable for the university as a whole. 


\section{A. Subjects}

The students were divided into two groups in order to assist with comparisons.

The 'conventional' students used the paper-based model, communicating with their instructors by mail or telephone, or in person at FTF practical and problem sessions. Paper assignments were exchanged by mail: the administrative protocol was managed using a multi-part cover sheet completed by the student, tutor, and university administrators at the appropriate stage through this process.

The Internet students communicated with their instructors and fellow students via electronic mail and HyperNews (our Web-based conferencing system). Practical and problem sessions, (tutorials), were conducted through asynchronous computer conferencing and e-mail. Student assignments were electronically documents sent as attachments to an automated handler. These were routed to the instructor who marked and returned them electronically.

\section{B. The Questionnaire}

At the beginning of the course a questionnaire was sent to conventional students via post and to Internet students via electronic mail. The questionnaire was piloted originally in 1995 with the initial 30 Internet students and was refined in later years. It asks students for their opinions on the model that they used. There are six sections:

\begin{tabular}{|l|l|l|}
\hline Section & Category & Type of Information \\
\hline 1 & Computer Use & $\begin{array}{l}\text { Questions on length and nature of experience, and on } \\
\text { student's self-assessment of ability, competence and } \\
\text { confidence }\end{array}$ \\
\hline 2 & E-mail and Network Use & $\begin{array}{l}\text { Questions on experience of, familiarity with, and current } \\
\text { usage of electronic mail, networks, and the Internet }\end{array}$ \\
\hline 3 & Programming & $\begin{array}{l}\text { Questions on length and depth of experience; self-assessment } \\
\text { of ability and confidence; familiar languages } \\
\text { applications; preferences }\end{array}$ \\
\hline 5 & Education and Employment & $\begin{array}{l}\text { Questions on educational and employment history, as well as } \\
\text { unpaid occupations and hobbies }\end{array}$ \\
\hline 6 & Style of Studying & Questions on study habits, patterns, and preferences. \\
\hline
\end{tabular}

Figure 1. Profile of Background Questionnaire.

\section{What did the Questionnaire Reveal?}

Conventional students reported limited interaction with fellow students and their tutor (about 5 times on average) and an inability to attend practical and problem sessions, due either to geographic location or work patterns. These practical sessions are reported by those who do attend as "useful for maintaining motivation and enthusiasm" and attendance at such is desirable both from a university and student perspective. In contrast, Internet students had higher participation in all types of interaction (contacting their tutor on average 20 times), their participation in practical sessions was higher: geographic and time barriers were evidently diminished. They reported enhancements such as increased flexibility and faster feedback. 
An underlying expectation of an Internet course reported by conventional students is that "e-mail is answered more quickly" and assignments are returned faster. Internet students corroborated this and reported that "e-mail queries were responded to very quickly and assignments were returned promptly".

Internet students were asked if they felt that they had learned more in this environment than in a conventional environment. No student felt they had learned more in terms of the content of the course, but they had gained valuable experience in using the Internet and its associated tools: "I have learned more about e-mail and I have greater confidence using the web. While this was not an objective of the course, per se, it is a welcome by-product of the Internet-based implementation.

We were concerned that technical aspects of an Internet-based course may have affected student experience negatively. Students were asked to describe their technical problems, but few were able to recall what the problems were. One student characterised the reason for this: "problems cease to be problems when they are solved." Fast and empathic problem solving is an important aspect of a positive student experience.

Internet communication has advantages for the student, but it also has raised student expectations - faster replies to e-mail and improved assignment turn-around time. This is an expectation we need to acknowledge when using such technologies.

\section{The Effect of the Transformed Learning Environment on Distance Students}

The rapid response to both queries and assignments are factors Internet students reported as enhancing their experience. They had expectations as to electronic communication and in this study they were met. Internet students stated that they would take another electronic course and reported the reasons as "increased flexibility and faster response time." While no student felt they learned more in relation to course content, they did feel their knowledge of electronic communication tools and techniques was greatly enhanced as was their confidence. Students reported gains as added value supplied by the Internet-based implementation.

\section{SUMMARY}

The accruals for distance education students who are geographically remote from tutor and fellow students are realised in the flexibility and rapidity of response. However these gains are counterbalanced by student expectations of faster and more efficient institutional practices. While the Internet-based implementation had significant gains for students there is impact on the university as a whole. "The task is not simply to understand an educational situation but to change it". The change, to be effective, needs to be more than a translation of an existing practice into another medium. We argue here that in order to provide an improved Internet-based system, an analysis of the system from the student perspective, is required. However there are considerations for the institution:

- Changing working practices from paper to Internet requires a change in culture.

- Initial investment (not readily available) is required to train staff in new roles and subsume electronic tasks into normal working practices.

- Culture and knowledge need time to spread. 
- More traditional students may feel intimidated by an unfamiliar medium.

- Students may feel disadvantaged by not having the most up-to-date equipment.

However balancing these costs are the benefits for students:

- Faster and more flexible access to information

- Faster registration and course enrolment

- Faster turnaround of assignments, enabling faster remedial action for learning

- Improved ability to submit assignments from anywhere--geography is not a barrier

- Increased interaction with tutor and fellow students

- More time to reflect on learning difficulties during the interactions

- Diminished time barriers to communication

- More supportive student-centred learning

- Reduced barriers of remoteness

The institution has now processed the experiences from this work and has developed an Internetbased implementation that will support students in all courses. The message of the work is that, in supporting distance education students electronically, those who fail to investigate the needs of their students thoroughly are likely to take poor advantage of new media and present a mediocre product.

\section{REFERENCES}

1. Adamson-Macedo, C., PCs, the Bandwidth Revolution and Engineering Curricula. IEE Computing and Control Engineering Journal, Vol. 7, No 3, pp. 129-135, 1996.

2. Steeples, C., Unsworth, C.,Bryson, M., Goodyear, P., Riding, P., Fowell, S., Levy, P., Duffy, C., Technological Support for Teaching and Learning: Computer-Mediated Communications in Higher Education (CMC in HE), Computer \& Education 26(1-3): 71-80, 1996.

3. Reinhardt, A., New Ways to Learn. Byte, Vol. 20, No. 3, pp. 55-71, 1995.

4. Skillicorn, D.B., Using Distributed Hypermedia for Collaborative Learning in Universities. Computer Journal, Vol. 36, No 6, pp. 471-482, 1996.

5. Thomas, P., Carswell, L., Emms, J., Petre, M., Poniatowska, B., Price, B., Distance Education Over the Internet. ACM SIGCSE/SIGCUE Proceedings, SIGCSE/SIGCUE Conference on Introducing Technology into Computer Science Education, Barcelona, ITiCSE ‘96, 1996.

6. Carswell, L., The "Virtual University": Toward an Internet Paradigm? Changing the Delivery of Computer Science Education, ACM SIGCSE/SIGCUE Proceedings, Dublin, Ireland, ITiCSE '98, 1998.

7. Jones, D., Computing by Distance Education, ACM SIGCSE Bulletin (Special Issue), 28, pp. 139-146, 1996.

8. Owen, G.S., Integrating World Wide Web Technology into Undergraduate Education ACM SIGCSE Bulletin (Special Issue), 28, pp. 101-103, 1996.

9. Pitt, M., The Use of Electronic Mail in Undergraduate Teaching. British Journal of Educational Technology, Vol. 27, No. 1, pp. 45-50,1996.

10. Rounder, D., Educational Technology in Curriculum Development. 2nd ed., Harper \& Row, 1982

\section{ACKNOWLEDGEMENTS}

With thanks to the MZX team, MZX instructors and students, Assignment Handling and Assignment Records Offices staff, and Statistical Support Services. 


\section{ABOUT THE AUTHORS}

Linda Carswell (l.carswell@open.ac.uk) is an experienced computing educator. Her Lecturship at the Open University is a special post concerned with exploiting technology in teaching. Her doctoral research tries to accommodate individual differences in teaching Computing concepts. She has an M.Sc. in Computer Science Education from the University of Ulster.

Dr. Pete Thomas (p.g.thomas@open.ac.uk), Ph.D. , is a Senior Lecturer at the Open University. His research interests are in Computer Science education, in particular electronic tuition, in which he has directed several projects concerned with the exploitation of the Internet for teaching. His current projects include audio-graphic tutorials and "AESOP: an electronic student observatory" for teaching programming and discovering how students learn. He has written several textbooks on programming languages, the most recent being Object Oriented Programming in Eiffel. His teaching interests include programming languages, operating systems, and the mathematical foundations of Computer Science.

Marian Petre (m.petre@open.ac.uk) is Senior Research Fellow and Director of the Centre for Informatics Education Research (CIER) at the Open University. CIER aims to integrate research and teaching objectives for both theoretical and practical issues in Computing education. Dr. Petre's personal research is centered on empirical studies (from observation to laboratory experiment) of how experts and novices actually structure information and use tools (including notations and other representations) in order to solve problems and design systems. Her Ph.D. in Computer Science is from University College London; her B.A. in Psycholinguistics is from Swarthmore College.

Blaine A. Price (B.A.Price@open.ac.uk) is a graduate of Queen's University (Canada) and the University of Toronto. He is a Lecturer in Computing at the Open University and is currently seconded to the OU's Knowledge Media Institute as their Chief Systems Strategist. His research areas include software visualization, empirical studies of programmers, and educational applications of Internet technology, especially in relation to Computer Science education. He designed and implemented much of the OU's prototype infrastructure for delivering courses via the Internet.

Mike Richards (M.Richards@open.ac.uk) is a graduate of the University of Wales (Aberystwyth). He is a project officer at the Open University specializing in Web applications and support of online distance learning students. His specialization is the design and implementation of web-based teaching materials. 